\title{
Astragaloside protects myocardial cells from apoptosis through suppression of the TLR4/NF-кB signaling pathway
}

\author{
YANG ZHAO ${ }^{1 *}$, ZHONGFEN LIU $^{2 *}$ and $\mathrm{HU}^{2} \mathrm{ZHANG}^{3}$ \\ ${ }^{1}$ Department of Cardiology, The First People's Hospital of Jinan, Jinan, Shandong 250000; \\ ${ }^{2}$ Department of Emergency Medical, The People's Hospital of Zhangqiu, Zhangqiu, Shandong 250200; \\ ${ }^{3}$ Department of Cardiovascular Surgery, The First Affiliated Hospital \\ of Kunming Medical University, Kunming, Yunnan 650032, P.R. China
}

Received July 24, 2017; Accepted November 8, 2017

DOI: $10.3892 / \mathrm{etm} .2017 .5535$

\begin{abstract}
Astragaloside is a monomer isolated from Astragalus membranaceus, a flowering plant in the family Fabaceae. The aim of the present study was to investigate the anti-apoptotic affect of astragaloside on myocardial cells through the TLR4/ $\mathrm{NF}-\kappa \mathrm{B}$ signaling pathway. Astragaloside, $\mathrm{NF}-\kappa \mathrm{B}$ inhibitor pyrrolidine dithiocarbamate (PDTC) and Toll-like receptor 4 (TLR4) blocking antibody solution were prepared in vitro, and myocardial cells were incubated and cultured in serumfree medium overnight. Cells were divided into five groups: the normal control group, serum-free group, astragaloside group, TLR4 blocking antibody group and NF- $\kappa \mathrm{B}$ inhibitor PDTC group. The myocardial cell apoptosis in each group was detected using flow cytometry, and the expression levels of TLR4 and NF- $\mathrm{NB}$ were detected via western blotting. The apoptosis rate in the serum-free group was significantly higher than that in the normal control group. The apoptosis rate of myocardial cells in the TLR4 blocking antibody group and $\mathrm{NF}-\kappa \mathrm{B}$ inhibitor PDTC group was lower than that in the serum-free group. In addition, the myocardial cell apoptosis was more obviously decreased in the astragaloside group, and the protein expression levels of TLR4 and NF- $\kappa \mathrm{B}$ in the serum-free group were significantly higher than those in normal control group. The protein expression levels of TLR4 and $\mathrm{NF}-\kappa \mathrm{B}$ in the astragaloside group were obviously lower than those in the serum-free group, and the protein expression levels of TLR4 and NF- $\mathrm{NB}$ in the TLR4 blocking antibody group and $\mathrm{NF}-\kappa \mathrm{B}$ inhibitor PDTC group were decreased. In conclusion, astragaloside reduced myocardial cell apoptosis and protected myocardial cells, which may be one of the mechanisms of a traditional Chinese medicine monomer in treating heart failure.
\end{abstract}

Correspondence to: Dr Hu Zhang, Department of Cardiovascular Surgery, The First Affiliated Hospital of Kunming Medical University, Kunming, Yunnan 650032, P.R. China

E-mail: zhanghu668@163.com

*Contributed equally

Key words: astragaloside, TLR4, NF-кB, myocardial cells

\section{Introduction}

Myocardial remodeling is a basic link in the occurrence and development of heart failure, and myocardial cell apoptosis, as one of the main causes of myocardial remodeling, promotes the developmental process of heart failure (1). Therefore, one of the targets for the effective prevention and treatment of heart failure is the inhibition of myocardial cell apoptosis. Toll-like receptot 4 (TLR4) belongs to the Toll-like receptor family, which is expressed in a variety of cells in the body, such as myocardial (2), vascular (3) and immune cells (4). Some scholars have found that TLR 2 can activate $\mathrm{NF}-\kappa \mathrm{B}$ expression in myocardial cells of SD rats, ultimately inducing myocardial cell apoptosis. However, TLR4 activation inhibits myocardial cell apoptosis (5). Astragalus membranaceus is a flowering plant in the family Fabaceae, and is one of the 50 fundamental herbs used in traditional Chinese medicine, Tonifying Qi. Astragaloside is a monomer derived from Astragalus membranaceus. Studies have found that astragaloside can effectively improve myocardial nutrition and myocardial cell mitochondria, which can also effectively inhibit TNF- $\alpha$-induced nuclear translocation of NF- $\kappa \mathrm{B}(6,7)$. In the present study, myocardial cells in normal growth were pretreated with astragaloside, $\mathrm{NF}-\kappa \mathrm{B}$ inhibitor pyrrolidine dithiocarbamate (PDTC) and TLR4 blocking antibody. The myocardial cell apoptosis was detected using a flow cytometer after the cells were cultured in serum-free medium, and the expression levels of TLR4 and $\mathrm{NF}-\kappa \mathrm{B}$ were detected via western blotting, so as to provide a theoretical basis for the application of astragaloside in the clinical treatment of heart failure.

\section{Materials and methods}

Experimental reagents. Dulbecco's modified Eagle's medium (DMEM) and fetal bovine serum (FBS) were purchased from Shanghai ScienCell Biotechnology Co., Ltd.). A cell apoptosis detection kit was obtained from Shanghai Yanhui Biotechnology Co., Ltd. (Shanghai, China), and $\mathrm{NF}-\kappa \mathrm{B}$ inhibitor PDTC was supplied by Beijing Baiaolaibo Technology Co., Ltd. (Beijing, China). TLR4 blocking solution was purchased from Cell Signaling Technology, and astragaloside was from Wuhan Yuancheng Technology 
Development Co., Ltd. (Wuhan, China). NE-PER cytoplasm and nuclear protein extraction kit was from Guangzhou Huayue Reacon Science Equipment Co., Ltd. (Guangzhou, China), and the Bradford protein quantification kit was purchased from Shanghai Genmed Pharmaceutical Science Co., Ltd. (Shanghai, China). Mouse anti-TLR4 antibody (cat. no. ABP57210; AmyJet Scientific Inc., Wuhan, China), and rabbit anti-NF- $\mathrm{KB}$ antibody and $\beta$-actin (cat. nos. 3045 and 4970; Cell Signaling Technology, Inc., Beverly, MA, USA) were used in the present study.

Culture, identification and grouping of myocardial cells Culture. Frozen tubes containing the myocardial cells were quickly placed on a floating plate and thawed via a water bath at $37^{\circ} \mathrm{C}$. Cells were removed from the frozen tubes when some ice was left, and culture solution was added, followed by centrifugation at $2,000 \mathrm{xg}$ for $5 \mathrm{~min}$ at $4^{\circ} \mathrm{C}$. Then the supernatant was discarded, and cells were resuspended and cultured.

Identification. Myocardial cells in the logarithmic growth phase were collected, washed with phosphate-buffered saline (PBS) once and fixed with $75 \%$ pre-cooled alcohol. After being washed with PBS 3 times, cells were permeabilized with $0.3 \%$ Triton X-100 for 30 min, washed again with PBS 3 times, and sealed at room temperature for $1 \mathrm{~h} . \alpha$-sarcomeric actin monoclonal antibody was used to incubate the cells overnight at $4^{\circ} \mathrm{C}$, and then cells were washed with PBS 3 times, incubated with $\operatorname{IgG}$ for $1 \mathrm{~h}$ and sealed using anti-fluorescent quenching liquid, immediately followed by observation under an inverted microscope.

Grouping. Astragaloside, NF- $\mathrm{kB}$ inhibitor PDTC and TLR4 blocking antibody solution were prepared in vitro. Myocardial cells were incubated and then cultured in serum-free medium overnight. Cells were divided into five groups: normal control group, serum-free group, astragaloside group, TLR4 blocking antibody group and NF- $\mathrm{KB}$ inhibitor PDTC group.

Detection of myocardial cell apoptosis via Annexin V-FITC-PI double-staining method. The detection of apoptotic cells was performed in strict accordance with the instructions included in the apoptosis detection kit. After the treatment of cells in the five groups, the cells were digested with trypsin and centrifuged at $2000 \mathrm{rpm}$ for $5 \mathrm{~min}$. The supernatant was then discarded and the cells were resuspended using $400 \mu \mathrm{l} 1 \mathrm{X}$ Annexin V binding solution; $5 \mu$ l Annexin V-FITC staining solution was added to incubate cells at $4^{\circ} \mathrm{C}$ in a dark place for $15 \mathrm{~min}$; then $10 \mu \mathrm{l}$ propidium iodide staining solution was added for reaction at room temperature in a dark place for 5 min, followed by detection using a flow cytometer.

Detection of TLR4 and NF- $\kappa B$ protein expression levels via western blotting. The total protein was extracted according to the instructions provided in the total protein extraction kit, and the concentration of the protein extracted was determined. Then the protein was stored at $-70^{\circ} \mathrm{C}$ for standby application. Gel with different concentrations was prepared for SDS polyacrylamide gel electrophoresis (PAGE). The gel positions of the two types of protein were selected according to the marker band, and the protein was transferred for $35 \mathrm{~min}$ after PVDF membrane transfer, sealed with $5 \%$ skim milk powder at $37^{\circ} \mathrm{C}$ for $90 \mathrm{~min}$, and incubated with the primary antibody (TLR4,
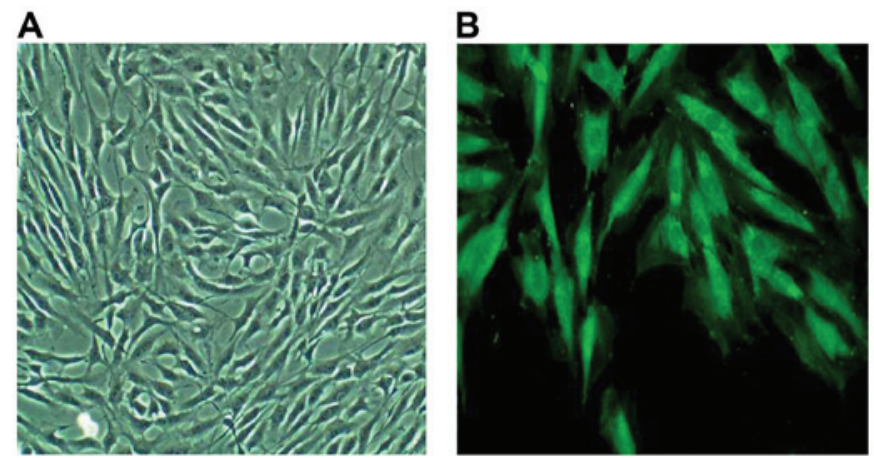

Figure 1. Morphology of myocardial cells cultured under the ordinary optical microscope (x200). Myocardial cells show the short column shape under an optical microscope (A), and green fluorescence under an inverted microscope (B).

NF- $\kappa B$ and $\beta$-actin; dilution, $1: 500$ ) at $4^{\circ} \mathrm{C}$ overnight; then Tris buffered saline Tween (TBST) was added and the mixture was vibrated on a shaking table 3 times ( $15 \mathrm{~min} / \mathrm{time})$. The protein was incubated with the secondary antibody at $37^{\circ} \mathrm{C}$ for $1 \mathrm{~h}$, then TBST was added again and the mixture was vibrated on a shaking table for $15 \mathrm{~min}$ (the operation was repeated 3 times); ECL solution was added and the protein was left in the dark room for exposure, development and fixation. Finally, the protein was scanned using the ChemiDoc ${ }^{\mathrm{TM}}$ MP imaging system. The image was analyzed using the professional image analysis software ImageJ [National Institutes of Health (NIH) Bethesda, MD, USA] followed by recording of the absorbance value.

Statistical analysis. The professional statistical software Statistical Product and Service Solutions (SPSS) 17.0 (provided by Beijing Xinmeijiahong Technology Co., Ltd., Beijing, China) was used for data analysis in this experiment. Data are presented as mean \pm standard deviation, and one-way analysis of variance was used for comparison among groups. $\mathrm{P}<0.05$ was considered to indicate a statistically significant difference.

\section{Results}

Myocardial cell culture. Myocardial cells that were cultured exhibited a short column shape under an optical microscope, as shown in Fig. 1A. Myocardial cells were identified using the $\alpha$-sarcomeric actin monoclonal immunofluorescence staining method, as shown in Fig. 1B.

Effect of serum-free culture on myocardial cell apoptosis. Myocardial cell apoptosis was detected using a flow cytometer via the Annexin V-FITC-PI double-staining method. There were few apoptotic myocardial cells in the normal control group. The apoptosis rate in the serum-free group was significantly higher than that in the normal control group and the difference was extremely significant $(\mathrm{P}<0.001)$. The apoptosis rate of myocardial cells in the TLR4 blocking antibody group was lower than that in the serum-free group, and the apoptosis rate of myocardial cells in the NF- $\mathrm{kB}$ inhibitor PDTC group was also lower than that in the serum-free group, and the differences were extremely significant $(\mathrm{P}<0.001)$. The apoptosis was 

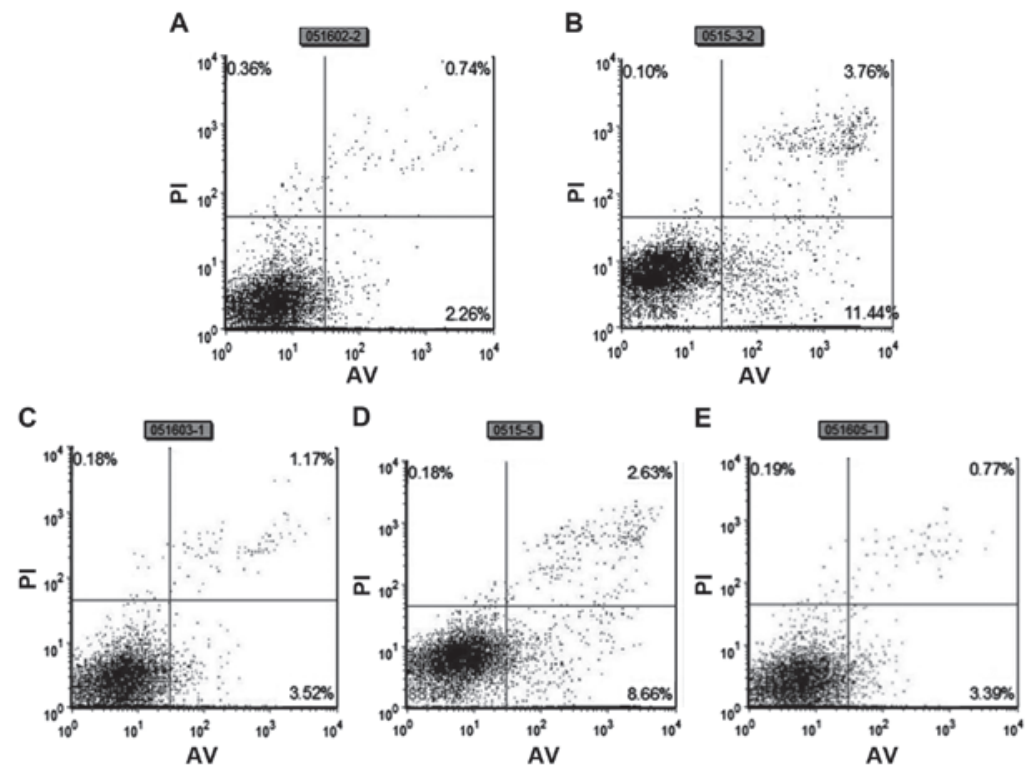

Figure 2. Detection of the myocardial cell apoptosis rate via Annexin V-FITC-PI double-staining method. Normal control group (A); serum-free group (B); astragaloside group (C); TLR4 blocking antibody group (D); PDTC group (E).

Table I. Myocardial cell apoptosis rates in the five groups (mean \pm SD).

\begin{tabular}{lccccr}
\hline Rate & Control group & Serum-free group & Astragaloside group & TLR4-Ab & PDTC \\
\hline Apoptosis rate $(\%)$ & $0.61 \pm 0.59$ & $4.29 \pm 1.25^{\mathrm{a}}$ & $0.98 \pm 0.31^{\mathrm{d}}$ & $2.36 \pm 0.27^{\mathrm{b}}$ & $1.94 \pm 0.73^{\mathrm{c}}$
\end{tabular}

${ }^{\mathrm{a}} \mathrm{P}<0.001$, compared with the control group. ${ }^{\mathrm{b}} \mathrm{P}<0.05,{ }^{\mathrm{c}} \mathrm{P}<0.001,{ }^{\mathrm{d}} \mathrm{P}<0.001$, compared with the serum-free group.

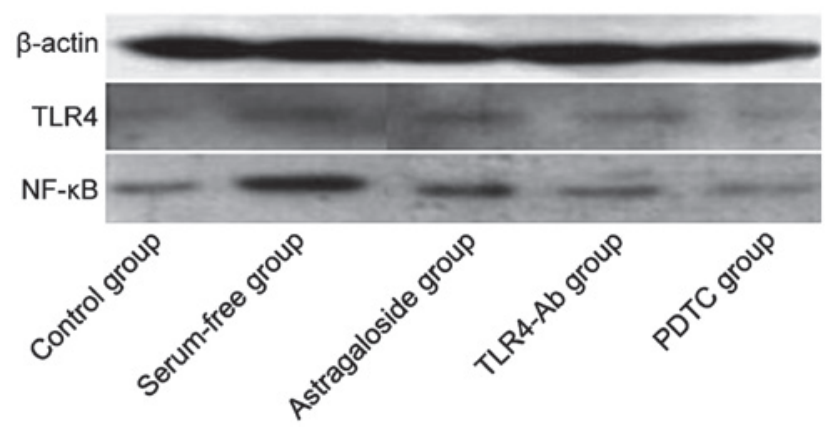

Figure 3. Western blot analysis of TLR4 and NF-אB p50 in myocardial cells in the five groups. Lane 1, control group; lane 2, serum-free group; lane 3, astragaloside group; lane 4, TLR4-Ab group; lane 5, PDTC group. TLR4, Toll-like receptor 4.

Table II. TLR4 and NF- $\mathrm{kB}$ p50 protein expression levels (mean $\pm \mathrm{SD}$ ).

\begin{tabular}{lccccc}
\hline Variables & Control group & Serum-free group & Astragaloside group & TLR4-Ab & PDTC \\
\hline TLR4/ $\beta$-actin & $0.3894 \pm 0.0491$ & $0.9024 \pm 0.0097^{\mathrm{a}}$ & $0.5845 \pm 0.1537^{\mathrm{b}}$ & $0.5238 \pm 0.1662^{\mathrm{b}}$ & $0.4839 \pm 0.1483^{\mathrm{b}}$ \\
p50/ $\beta$-actin & $0.5016 \pm 0.0593$ & $1.1038 \pm 0.0739^{\mathrm{a}}$ & $0.7585 \pm 0.2016^{\mathrm{b}}$ & $0.6048 \pm 0.1529^{\mathrm{b}}$ & $0.4982 \pm 0.0591^{\mathrm{b}}$
\end{tabular}

${ }^{\mathrm{a}} \mathrm{P}<0.001$, compared with the control group; ${ }^{\mathrm{b}} \mathrm{P}<0.05$, compared with the serum-free group. TLR 4 , Toll-like receptor 4.

alleviated in the astragaloside group, and the difference was extremely significant $(\mathrm{P}<0.001)$ (Fig. 2 and Table I).
Detection of TLR4 and NF- $\mathrm{B}$ protein expression levels via western blotting. The TLR4 and NF-KB protein expression 
levels were analyzed via western blotting. The protein expression levels of TLR4 and NF- $\kappa \mathrm{B}$ in the serum-free group were significantly higher than those in the normal control group and the differences were extremely significant. The protein expression levels of TLR4 and NF- $\mathrm{NB}$ in the astragaloside group were significantly lower than those in the serum-free group, and the protein expression levels of TLR4 and NF- $\mathrm{BB}$ in the TLR4 blocking antibody group and NF- $\kappa \mathrm{B}$ inhibitor PDTC group were decreased (Fig. 3 and Table II).

\section{Discussion}

The final stage of various heart diseases usually leads to heart failure $(8,9)$. Once heart failure occurs, myocardial cell apoptosis results, and apoptotic myocardial cells will further accelerate the process of heart failure at the same time (10-12). The defects of myocardial cells can be reduced and the occurrence and development of heart failure can be alleviated by investigating the molecular mechanism of myocardial cell apoptosis, by inhibiting the signal transduction pathway of apoptosis and by increasing the anti-apoptosis capacity of cells. One of the research 'hotspots' in recent years is improvement of the heart function after heart failure by reducing myocardial cell apoptosis. In the present study, using Annexin V-FITC-PI double-staining method, it was found that $4.29 \%$ of myocardial cells were apoptotic after being cultured under serum-free conditions for $24 \mathrm{~h}$ compared to $0.98 \%$ in the astragaloside treatment group.

TLR4 is one of the members of the Toll-like receptor family. Many scholars in China and foreign countries have confirmed that TLR4 binds to ligands, activates NF- $\kappa \mathrm{B}$ and increases myocardial cell apoptosis through many signaling pathways in the in vivo signal transduction process $(13,14)$. The application of TLR4 blocking antibody or NF- $\kappa \mathrm{B}$ inhibitor can significantly reduce myocardial cell apoptosis (15). Therefore, it is speculated that the TLR4/NF- $\kappa \mathrm{B}$ signaling pathway is closely related to myocardial cell apoptosis.

The serum-free cell model was used in this study to activate the TLR4 signal transduction pathway in myocardial cells. The myocardial cells were pretreated with TLR4 blocking antibody and NF- $\mathrm{NB}$ inhibitor PDTC, and then cultured in serum-free medium for $24 \mathrm{~h}$. The experimental results showed that the TLR4 signal transduction pathway in myocardial cells was activated after serum-free culture for $24 \mathrm{~h}$. Flow cytometry showed that there were more apoptotic myocardial cells, and the TLR 4 and $\mathrm{NF}-\kappa \mathrm{B}$ protein expression levels were also increased significantly. In the TLR4 blocking antibody group, the number of apoptotic myocardial cells was decreased obviously, and the TLR 4 and $\mathrm{NF}-\kappa \mathrm{B}$ protein expression levels were also decreased. At the same time, inhibition of TLR4 protein expression inhibited the NF- $\kappa$ B protein expression, while the inhibition of $\mathrm{NF}-\kappa \mathrm{B}$ protein expression also inhibited the TLR4 protein expression, indicating that there is a positive feedback regulatory mechanism between the TLR4 and NF- $\kappa \mathrm{B}$ protein expression levels. The number of apoptotic myocardial cells was significantly decreased after they were pretreated with TLR4 blocking antibody and NF- $\kappa \mathrm{B}$ inhibitor PDTC. Therefore, it was confirmed in this study that TLR4/ $\mathrm{NF}-\kappa \mathrm{B}$ signal transduction pathway is related to the apoptosis of myocardial cells.
Astragalus membranaceus is a flowering plant in the family Fabaceae, and is one of the 50 fundamental herbs used in traditional Chinese medicine, Tonifying Qi. Astragaloside is one of the active ingredients derived from Astragalus membranaceus. Studies have found that astragaloside can effectively improve myocardial nutrition and myocardial cell mitochondria, which can also effectively inhibit the NF- $\mathrm{NB}$ nuclear translocation function. In this study, myocardial cells were pretreated with astragaloside using the same methods as TLR4 blocking antibody and $\mathrm{NF}-\kappa \mathrm{B}$ inhibitor PDTC. The myocardial cell apoptosis rate was detected using a flow cytometer and the protein expression levels of TLR4 and NF- $\mathrm{NB}$ were detected via western blotting. The results showed that astragaloside induced the apoptosis of myocardial cells and reduced the expression levels of TLR4 and NF- $\kappa \mathrm{B}$ proteins in myocardial cells induced by serum-free medium. Thus, we speculate that astragaloside has a significant inhibitory effect on the apoptosis of myocardial cells, and the possible molecular mechanism is that this traditional Chinese medicine itself can inhibit the expression of TLR4 protein in myocardial cells, or inhibit various cytokines in the downstream of $\mathrm{NF}-\kappa \mathrm{B}$. Moreover, these cytokines have a positive feedback regulatory effect on TLR4 in myocardial cells induced by serum-free medium, which can stimulate the expression of TLR4. When these cytokines are inhibited by astragaloside, the activation of TLR4 is reduced, thus reducing the expression of TLR4. The specific inhibition mechanism remains to be further verified through further experiments.

In the present study, the apoptosis of myocardial cells in the five groups was detected via flow cytometry, and the protein expression levels of TLR 4 and $\mathrm{NF}-\kappa \mathrm{B}$ in myocardial cells in the five groups were detected via western blotting. The results showed that astragaloside significantly reduced myocardial cell apoptosis and effectively protected the myocardial cells. This may be one of the mechanisms of a traditional Chinese medicine monomer in the treatment of heart failure, providing a theoretical basis for the application of astragaloside in the clinical treatment of heart failure.

\section{References}

1. Vallejo JG: Role of toll-like receptors in cardiovascular diseases. Clin Sci (Lond) 121: 1-10, 2011.

2. Krishnan J, Selvarajoo K, Tsuchiya M, Lee G and Choi S: Toll-like receptor signal transduction. Exp Mol Med 39: 421-438, 2007.

3. Zhang HY, Kang J, Han WJ, Hu MM and Jia HG: The expression and significance of TLR4, MyD88 and NF- $\mathrm{BB}$ mRNA in mouse lymph node of experimental autoimmune myositis. Xi Bao Yu Fen Zi Mian Yi Xue Za Zhi 28: 272-275, 2012 (In Chinese).

4. Liu Q, Zhang J, Xu Y, Huang Y and Wu C: Effect of carvedilol on cardiomyocyte apoptosis in a rat model of myocardial infarction: A role for toll-like receptor 4. Indian J Pharmacol 45: 458-463, 2013.

5. Watari K, Nakaya M, Nishida M, Kim KM and Kurose $H$ : $\beta$-arrestin 2 in infiltrated macrophages inhibits excessive inflammation after myocardial infarction. PLoS One 8: e68351, 2013.

6. Liu CC, Huang Y, Zhang JH, Xu Y and Wu CH: Effect of carvedilol on cardiac dysfunction 4 days after myocardial infarction in rats: Role of toll-like receptor 4 and $\beta$-arrestin 2 . Eur Rev Med Pharmacol Sci 17: 2103-2110, 2013.

7. Liu J, Chen Q, Jian Z, Xiong X, Shao L, Jin T, Zhu X and Wang L: Daphnetin protects against cerebral ischemia/reperfusion injury in mice via inhibition of TLR4/NF- $\kappa \mathrm{B}$ signaling pathway. BioMed Res Int 2016: 2816056, 2016. 
8. Song Y, Hou M, Li Z, Luo C, Ou JS, Yu H, Yan J and Lu L: TLR4/NF- $\kappa$ B/Ceramide signaling contributes to Ox-LDLinduced calcification of human vascular smooth muscle cells. Eur J Pharmacol 794: 45-51, 2017.

9. Sun Y, Huang J and Song K: BET protein inhibition mitigates acute myocardial infarction damage in rats via the TLR4/ TRAF6/NF-кB pathway. Exp Ther Med 10: 2319-2324, 2015.

10. Trentin-Sonoda M, da Silva RC, Kmit FV, Abrahão MV, Monnerat Cahli G, Brasil GV, Muzi-Filho H, Silva PA, Tovar-Moll FF, Vieyra A, et al: Knockout of Toll-like receptors 2 and 4 prevents renal ischemia-reperfusion-induced cardiac hypertrophy in mice. PLoS One 10: e0139350, 2015.

11. Yang J, Wang HX, Zhang YJ, Yang YH, Lu ML, Zhang J, Li ST, Zhang SP and Li G: Astragaloside IV attenuates inflammatory cytokines by inhibiting TLR4/NF-кB signaling pathway in isoproterenol-induced myocardial hypertrophy. $\mathrm{J}$ Ethnopharmacol 150: 1062-1070, 2013.

12. Li M, Yu L, She T, Gan Y, Liu F, Hu Z, Chen Y, Li S and Xia H: Astragaloside IV attenuates Toll-like receptor 4 expression via $\mathrm{NF}-\kappa \mathrm{B}$ pathway under high glucose condition in mesenchymal stem cells. Eur J Pharmacol 696: 203-209, 2012.
13. Lu M, Tang F, Zhang J, Luan A, Mei M, Xu C, Zhang S, Wang H and Maslov LN: Astragaloside IV attenuates injury caused by myocardial ischemia/reperfusion in rats via regulation of toll-like receptor $4 /$ nuclear factor- $\kappa \mathrm{B}$ signaling pathway. Phytother Res 29: 599-606, 2015.

14. Zhang WJ and Frei B: Astragaloside IV inhibits NF- $\kappa$ B activation and inflammatory gene expression in LPS-treated mice. Mediators Inflamm 2015: 274314, 2015

15. He YX, Shi HL, Liu HS, Wu H, Zhang BB, Wu XJ and Wang ZT: Astragaloside IV regulates STAT1/I $\kappa \mathrm{B} / \mathrm{NF}-\kappa \mathrm{B}$ signaling pathway to inhibit activation of BV-2 cells. Zhongguo Zhong Yao Za Zhi 40: 124-128, 2015 (In Chinese).

(i) $\Theta$ This work is licensed under a Creative Commons Attribution-NonCommercial-NoDerivatives 4.0 International (CC BY-NC-ND 4.0) License. 